\title{
Regression of primary cardiac angiosarcoma and metastatic nodules following propranolol as a single agent treatment
}

\author{
Dana C. Galván', Anoop P. Ayyappan², Brad A. Bryan³ \\ ${ }^{1}$ Paul L. Foster School of Medicine, Texas Tech University Health Sciences Center, El Paso, TX, USA \\ ${ }^{2}$ Department of Radiology, Texas Tech University Health Sciences Center, El Paso, TX, USA \\ ${ }^{3}$ Department of Biomedical Sciences, Texas Tech University Health Sciences Center, El Paso, TX, USA \\ Correspondence to: Brad A. Bryan, email: brad.bryan@ttuhsc.edu \\ Keywords: angiosarcoma; cardiac tumor; metastatic; propranolol; beta blocker \\ Received: May 15, 2018 \\ Accepted: August 2, 2018 \\ Published: October 11, 2018 \\ Copyright: Galván et al. This is an open-access article distributed under the terms of the Creative Commons Attribution License \\ 3.0 (CC BY 3.0), which permits unrestricted use, distribution, and reproduction in any medium, provided the original author and \\ source are credited.
}

\section{ABSTRACT}

Angiosarcoma is the most common malignant cardiac tumor. Cardiac angiosarcoma is a highly lethal neoplasm that is largely resistant to conventional anti-cancer therapy. Mean survival of patients with cardiac angiosarcoma is only 4 months, and almost all patients will succumb to the disease within 1 year. The beta blocker propranolol is an emerging therapy against angiosarcoma. When combined with conventional therapies, propranolol increases progression free and overall survival in patients with this tumor type. It is currently unknown if propranolol is capable of showing anti-cancer efficacy as a single agent therapy. We report a case of a 61 year old woman diagnosed with primary cardiac angiosarcoma and liver and lung metastases. This patient chose to decline conventional therapy, and instead was prescribed the beta blocker propranolol as a single agent treatment. After 12 months, the mediastinal mass substantially debulked and decreased in size, and the metastatic nodules stabilized or resolved with no evidence of hyper-metabolic activity on PET-CT. This is the first reported data showing long term efficacy of the beta blocker propranolol as a single agent therapy against angiosarcoma.

\section{INTRODUCTION}

Primary cardiac neoplasms are exceptionally rare tumors, with an autopsy incidence of $0.0001 \%$ to $0.03 \%$ [1]. The most common malignant cardiac tumor is angiosarcoma, an endothelial cell sarcoma that comprises approximately $2 \%$ of all primary cardiac neoplasms $[2,3]$. Cardiac angiosarcomas predominantly occur in males who are less than 65 years of age $[4,5]$, and have been shown, at least in some cases, to display a familial pattern of inheritance corresponding to a germline POT1 mutation [6,7]. Patient diagnosis is often delayed as a result of presenting vague, generalized symptoms that initially appear relatively benign including shortness of breath, weight loss, and fatigue associated with anemia [8]. A definitive diagnosis of angiosarcoma is generally confirmed using a combination of imaging, histology, and positive expression of vascular protein markers including
CD31 and CD34 [9-13]. Metastatic disease is very common at the time of diagnosis, with most disseminated tumors occurring in the liver, lymph nodes, bone, adrenal glands, and spleen [14].

Nearly $80 \%$ of cardiac angiosarcomas arise in the right atrium where they typically replace the atrial wall, fill the entire cardiac chamber, and aggressively infiltrate into the surrounding pericardium. Extensive pericardial spread and encasement of the heart occurs rapidly, leading to congestive heart failure, pericardial effusion, and cardiac tamponade. Surgical excision of the mass is the preferred treatment option, however the rarity of cardiac angiosarcoma has made treatment difficult to standardize and the benefit of adjunctive chemotherapy and/or radiation is currently unknown. Even with comprehensive therapy, mean survival is only $\sim 4$ months, and greater than $90 \%$ of patients die within 1 year of diagnosis [14-18].

Emerging evidence from a number of retrospective clinical analyses and prospective case reports indicates 


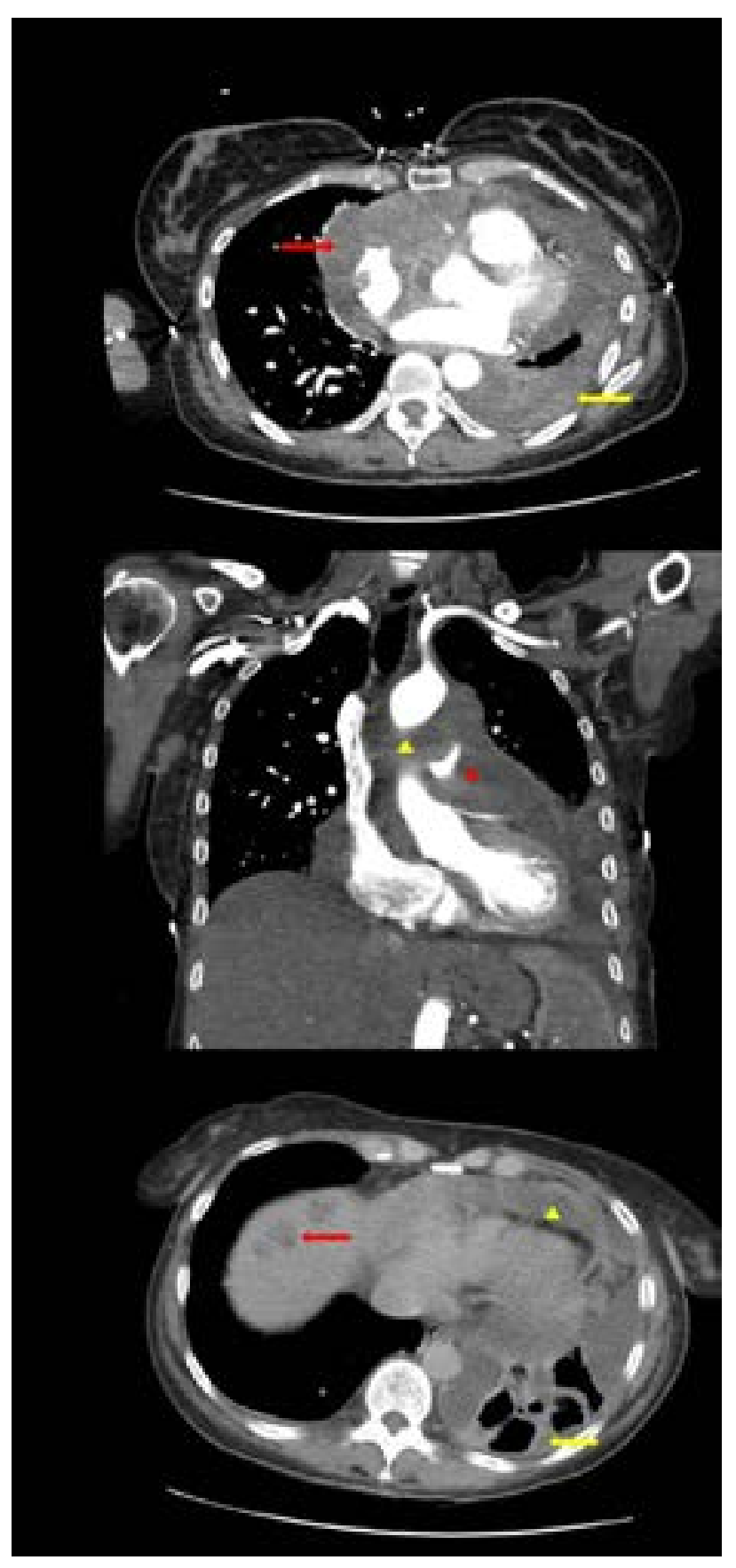

Figure 1: CT imaging of cardiac angiosarcoma prior to propranolol treatment. (A) Baseline axial chest CT showing a large soft-tissue mass invading the right atrium and encasing the heart (red arrow). There is also nodular pleural thickening (yellow arrow) along the costal and mediastinal pleural surfaces and a small pleural effusion secondary to pleural infiltration. (B) Baseline coronal chest CT shows soft tissue mass encasing the ascending aorta (yellow arrowhead) and main pulmonary artery (yellow arrowhead). (C) Baseline axial chest CT shows circumferential pericardial thickening and effusion (yellow arrowhead) and nodular pleural thickening (yellow arrow) with loculated pleural effusion. Note small hypodense lesions in the right lobe of the liver consistent with metastases (red arrow). that the beta blocker propranolol exhibits clinical efficacy against primary and metastatic angiosarcomas [19-26]. Due to a dearth of effective treatment options against angiosarcomas, and the remarkable clinical outcomes reported in the literature for propranolol, this beta blocker has recently received Orphan Drug Designation against angiosarcomas by the European Medicines Agency. Durable efficacy of propranolol against angiosarcomas has been reported when this drug is used in combination with conventional therapies such as surgery, chemotherapy, and/or radiation. To date, no studies have examined the long-term efficacy of propranolol as a single agent therapy against angiosarcoma.

\section{CASE PRESENTATION}

In late 2016, a 61 year old non-smoking female presented with exertional shortness of breath, mild pedal edema, distended neck veins, and a recent weight gain of $15 \mathrm{lbs}$ over the prior 2 weeks. It was initially suspected that the patient was experiencing pulmonary embolism based on her clinical presentation and elevated D-dimer, but further testing revealed that she was experiencing cardiac tamponade with mild pulmonary hypertension and heart failure. Pericardiocentesis was performed without definitive diagnosis. In February 2017, the patient exhibited worsening respiratory symptoms without fever, hemoptysis, sputum production, B symptoms, or extremity edema. Baseline chest PET-CT images were reviewed by radiologists at both UCLA Oncology and Texas Tech University Health Sciences Center, demonstrating a large middle mediastinal mass encasing the main pulmonary artery, with pericardial and left pleural effusion (Figure 1A \& Figure 1B). Scattered pulmonary nodules and hypodense lesions were identified in the right lobe of the liver, consistent with a diagnosis of metastatic disease (Figure 1C). Biopsy of the mediastinal mass revealed a high grade undifferentiated malignant neoplasm composed of highly proliferative (ki67 staining 50\%) pleomorphic anaplastic epithelioid malignant cells with large areas of necrosis and fibrosis. Immunohistochemistry revealed strong antigenicity for CD31 and CD34, and weak antigenicity for D2-40 and Factor VIII indicating a diagnosis of angiosarcoma that was corroborated at both UC San Diego Health and MD Anderson.

Conventional treatment options were recommended, however the patient declined these based on low reported survival rates, and instead, requested the non-selective beta blocker propranolol as a single agent therapy. In May $2017,40 \mathrm{mg} / \mathrm{kg}$ propranolol was administered daily and PET-CT scans were performed at regular intervals to assess the response of the tumor to propranolol. Assessment of tumor response was based on ${ }^{18} \mathrm{~F}$-fluorodeoxyglucose (FDG) tracer uptake and measurements/assessments of the primary tumor and distant metastases. After 12 months of propranolol as a single agent therapy, significant debulking 
and decreased size of the residual mediastinal mass was observed on PET-CT scans, with resolution of pericardial effusion (Figure 2A \& Figure 2B). Pulmonary nodules were stable to regressed, and the nodules in the right lobe of the liver had completely resolved (Figure 2C). There was no evidence of residual hyper-metabolic activity based on FDG measurements in the primary lesion or in metastatic sites in the chest, abdomen, or pelvis on PETCT.

\section{DISCUSSION}

The targets of propranolol, the beta adrenergic receptor proteins ( $\beta$-AR1, $\beta$-AR2, $\beta$-AR3), are expressed in angiosarcoma $[25,27]$. Preclinical studies using in vitro and in vivo angiosarcoma models indicate that propranolol as a single agent is capable of reducing tumor cell proliferation, selectively inducing tumor cell apoptosis while sparing non-diseased cell types, and inhibiting the growth of angiosarcoma xenografts in animal tumor models $[23,25,26]$. Translation of these findings into human patients revealed that propranolol alone is capable of reducing the angiosarcoma proliferative index after only 1 week of treatment [20], however the long term efficacy of propranolol as a single agent therapy was not individually evaluated in the aforementioned study, as the tumor was subsequently treated with radiation and chemotherapy. Nonrandomized prospective and retrospective studies have combined propranolol with conventional anti-sarcoma therapies, leading to substantially increased progression free- and overall-survival of angiosarcoma patients [23, 26], however randomized clinical studies are necessary to comprehensively evaluate the efficacy and optimal dosing of propranolol against angiosarcoma. Several targeted therapeutics such as Pazopanib, TRC105, and others are currently being tested clinically against this tumor type. Future studies should evaluate which specific combination of conventional or targeted therapeutics maximizes patient survival when combined with propranolol.

Strong efficacy of propranolol as a single agent against other vascular tumor types has been reported for infantile hemangioma [28, 29], Kaposi's sarcoma [30], hemangioendothelioma [31-33], and hemangioblastoma [34-36]. Regardless of convention therapy, almost all patients will succumb to cardiac angiosarcoma within a year of diagnosis [14-18], and for patients who cannot or choose not to undergo surgery, chemotherapy, and/ or radiation, supportive care has historically remained their only remaining option. The current data presented in this report suggests that propranolol may have antitumor efficacy against cardiac angiosarcomas. Use of propranolol may be a viable alternative for patients who

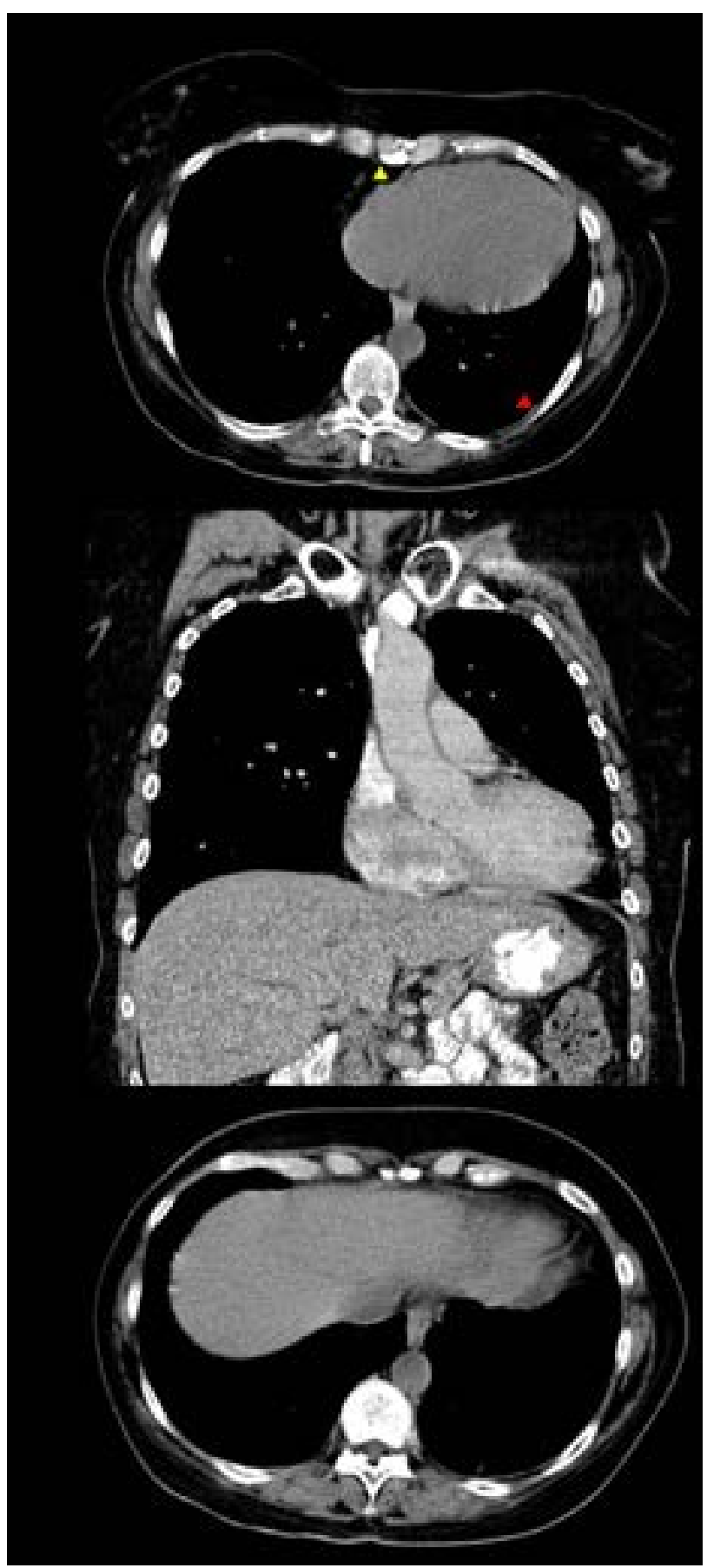

Figure 2: CT imaging of cardiac angiosarcoma subsequent to propranolol treatment. (A) Axial chest CT following treatment with non-selective beta blocker shows marked reduction in size of the pericardial and pleural mass. There is minimal residual pleural (red arrowhead) and pericardial thickening (yellow arrowhead). (B) Coronal chest CT shows regression of soft tissue mass encasing the ascending aorta and main pulmonary artery after treatment with non-selective beta blocker. (C) Axial CT abdomen following treatment with nonselective beta blocker shows resolution of liver metastases. 
elect to forego conventional therapy.

\section{CONFLICTS OF INTEREST}

The authors declare no potential conflicts of interest.

\section{FUNDING}

This study was supported by grants to $\mathrm{BAB}$ from Sarcoma Foundation of America and Angiosarcoma Awareness Foundation.

\section{REFERENCES}

1. Look Hong NJ, Pandalai PK, Hornick JL, Shekar PS, Harmon DC, Chen YL, Butrynski JE, Baldini EH, Raut CP. Cardiac angiosarcoma management and outcomes: 20year single-institution experience. Ann Surg Oncol. 2012; 19:2707-15.

2. Kupsky DF, Newman DB, Kumar G, Maleszewski JJ, Edwards WD, Klarich KW. Echocardiographic Features of Cardiac Angiosarcomas: The Mayo Clinic Experience (1976-2013). Echocardiography. 2016; 33:186-92.

3. Kurian KC, Weisshaar D, Parekh H, Berry GJ, Reitz B. Primary cardiac angiosarcoma: case report and review of the literature. Cardiovasc Pathol. 2006; 15:110-12.

4. Antonuzzo L, Rotella V, Mazzoni F, Doni L, Bianchini D, Garbini F, Maio V, Di Costanzo F. Primary cardiac angiosarcoma: a fatal disease. Case Rep Med. 2009; 2009:591512.

5. Hamidi M, Moody JS, Weigel TL, Kozak KR. Primary cardiac sarcoma. Ann Thorac Surg. 2010; 90:176-81.

6. Calvete $\mathrm{O}$, Martinez P, Garcia-Pavia P, Benitez-Buelga C, Paumard-Hernández B, Fernandez V, Dominguez F, Salas C, Romero-Laorden N, Garcia-Donas J, Carrillo J, Perona R, Triviño JC, et al. A mutation in the POT1 gene is responsible for cardiac angiosarcoma in TP53-negative LiFraumeni-like families. Nat Commun. 2015; 6:8383.

7. Casha AR, Davidson LA, Roberts P, Nair RU. Familial angiosarcoma of the heart. J Thorac Cardiovasc Surg. 2002; 124:392-94.

8. Brandt RR, Arnold R, Bohle RM, Dill T, Hamm CW. Cardiac angiosarcoma: case report and review of the literature. Z Kardiol. 2005; 94:824-28.

9. De Young BR, Frierson HF Jr, Ly MN, Smith D, Swanson PE. CD31 immunoreactivity in carcinomas and mesotheliomas. Am J Clin Pathol. 1998; 110:374-77.

10. Folpe AL, Chand EM, Goldblum JR, Weiss SW. Expression of Fli-1, a nuclear transcription factor, distinguishes vascular neoplasms from potential mimics. Am J Surg Pathol. 2001; 25:1061-66.

11. Folpe AL, Kwiatkowski DJ. Perivascular epithelioid cell neoplasms: pathology and pathogenesis. Hum Pathol. 2010;
41:1-15.

12. Heim-Hall J, Yohe SL. Application of immunohistochemistry to soft tissue neoplasms. Arch Pathol Lab Med. 2008; 132:476-89.

13. Pusztaszeri MP, Seelentag W, Bosman FT. Immunohistochemical expression of endothelial markers CD31, CD34, von Willebrand factor, and Fli-1 in normal human tissues. J Histochem Cytochem. 2006; 54:385-95.

14. Butany J, Yu W. Cardiac angiosarcoma: two cases and a review of the literature. Can J Cardiol. 2000; 16:197-205.

15. Janigan DT, Husain A, Robinson NA. Cardiac angiosarcomas. A review and a case report. Cancer. 1986; $57: 852-59$.

16. Keeling IM, Ploner F, Rigler B. Familial cardiac angiosarcoma. Ann Thorac Surg. 2006; 82:1576.

17. Pigott C, Welker M, Khosla P, Higgins RS. Improved outcome with multimodality therapy in primary cardiac angiosarcoma. Nat Clin Pract Oncol. 2008; 5:112-15.

18. Ram Prabu MP, Thulkar S, Ray R, Bakhshi S. Primary cardiac angiosarcoma with good response to Paclitaxel. J Thorac Oncol. 2011; 6:1778-79.

19. Banavali S, Pasquier E, Andre N. Targeted therapy with propranolol and metronomic chemotherapy combination: sustained complete response of a relapsing metastatic angiosarcoma. Ecancermedicalscience. 2015; 9:499.

20. Chow W, Amaya CN, Rains S, Chow M, Dickerson EB, Bryan BA. Growth Attenuation of Cutaneous Angiosarcoma With Propranolol-Mediated $\beta$-Blockade. JAMA Dermatol. 2015; 151:1226-29.

21. Daguzé J, Saint-Jean M, Dréno B. Large nose angiosarcoma treated effectively with oral cyclophosphamide combined with propranolol. J Eur Acad Dermatol Venereol. 2018; 32:e52-54.

22. Daguzé J, Saint-Jean M, Peuvrel L, Cassagnau E, Quéreux G, Khammari A, Dréno B. Visceral metastatic angiosarcoma treated effectively with oral cyclophosphamide combined with propranolol. JAAD Case Rep. 2016; 2:497-99.

23. Pasquier E, André N, Street J, Chougule A, Rekhi B, Ghosh J, Philip DS, Meurer M, MacKenzie KL, Kavallaris M, Banavali SD. Effective Management of Advanced Angiosarcoma by the Synergistic Combination of Propranolol and Vinblastine-based Metronomic Chemotherapy: A Bench to Bedside Study. EBioMedicine. 2016; 6:87-95.

24. Pramanik R, Gogia A, Malik PS, Gogi R. Metastatic Primary Angiosarcoma of the Breast: Can We Tame It the Metronomic Way. Indian J Med Paediatr Oncol. 2017; $38: 228-31$

25. Stiles JM, Amaya C, Rains S, Diaz D, Pham R, Battiste J, Modiano JF, Kokta V, Boucheron LE, Mitchell DC, Bryan BA. Targeting of beta adrenergic receptors results in therapeutic efficacy against models of hemangioendothelioma and angiosarcoma. PLoS One. 
2013; 8:e60021.

26. Amaya CN, Perkins M, Belmont A, Herrera C, Nasrazadani A, Vargas A, Khayou T, Montoya A, Ballou Y, Galvan D, Rivas A, Rains S, Patel L, et al. Non-selective beta blockers inhibit angiosarcoma cell viability and increase progression free- and overall-survival in patients diagnosed with metastatic angiosarcoma. Oncoscience. 2018; 5:109-19. doi: https://doi.org/10.18632/oncoscience.413

27. Chisholm KM, Chang KW, Truong MT, Kwok S, West RB, Heerema-McKenney AE. $\beta$-Adrenergic receptor expression in vascular tumors. Mod Pathol. 2012; 25:1446-51.

28. Léauté-Labrèze C, Dumas de la Roque E, Hubiche T, Boralevi F, Thambo JB, Taïeb A. Propranolol for severe hemangiomas of infancy. N Engl J Med. 2008; 358:264951.

29. Léauté-Labrèze C, Hoeger P, Mazereeuw-Hautier J, Guibaud L, Baselga E, Posiunas G, Phillips RJ, Caceres H, Lopez Gutierrez JC, Ballona R, Friedlander SF, Powell J, Perek D, et al. A randomized, controlled trial of oral propranolol in infantile hemangioma. N Engl J Med. 2015; 372:735-46.

30. McAllister SC, Hanson RS, Manion RD. Propranolol Decreases Proliferation of Endothelial Cells Transformed by Kaposi's Sarcoma-Associated Herpesvirus and Induces Lytic Viral Gene Expression. J Virol. 2015; 89:11144-49.

31. Filippi L, Tamburini A, Berti E, Perrone A, Defilippi C, Favre C, Calvani M, Della Bona ML, la Marca G, Donzelli G. Successful Propranolol Treatment of a Kaposiform Hemangioendothelioma Apparently Resistant to Propranolol. Pediatr Blood Cancer. 2016; 63:1290-92.

32. Maaloul I, Aloulou H, Hentati Y, Kamoun T, Mnif Z, Hachicha M. Infantile hepatic hemangioendothelioma successfully treated by low dose of propranolol. Presse Med. 2017; 46:454-56.

33. Özdemir ZC, Düzenli Kar Y, Şöhret NC, Kebapçı M, Bör Ö. Beta blocker and steroid therapy in the treatment of infantile hepatic hemangioendothelioma. Drug Discov Ther. 2017; 11:161-64.

34. Albiñana V, Escribano RM, Soler I, Padial LR, RecioPoveda L, Villar Gómez de Las Heras K, Botella LM. Repurposing propranolol as a drug for the treatment of retinal haemangioblastomas in von Hippel-Lindau disease. Orphanet J Rare Dis. 2017; 12:122.

35. Albiñana V, Villar Gómez de Las Heras K, Serrano-Heras G, Segura T, Perona-Moratalla AB, Mota-Pérez M, de Campos JM, Botella LM. Propranolol reduces viability and induces apoptosis in hemangioblastoma cells from von Hippel-Lindau patients. Orphanet J Rare Dis. 2015; 10:118.

36. Hernández-Martínez P, Gallego-Pinazo R, Dolz-Marco R, Arevalo JF, Díaz-Llopis M, Cisneros Lanuza A. Oral propranolol and intravitreal ranibizumab for refractory serous macular detachment secondary to retinal capillary hemangioblastoma. Arch Soc Esp Oftalmol. 2015; 90:399401. 